hep-th 9701126

\title{
Charged Open Membrane Solution On A Manifold With A Boundary
}

\author{
Fermin $\mathrm{ALDABE}^{1}$ and Arne L. LARSEN ${ }^{2}$ \\ January 24, 1997 \\ ${ }^{1}$ Theoretical Physics Institute, Department of Physics, \\ University of Alberta, Edmonton, Canada T6E 2J1 \\ email: faldabe@phys.ualberta.ca \\ ${ }^{2}$ Observatoire de Paris, DEMIRM, 61, Av. de l'Observatoire, \\ 75014 Paris, France \\ email: larsen@mesioa.obspm.fr
}

\begin{abstract}
Explicit open single and multi-membrane solutions of the low energy limit of M-theory on the orbifold $R^{10} \times$ $S^{1} / Z_{2}$ are presented. This low energy action is described by an 11dimensional supergravity action coupled to two $E_{8}$ super YangMills fields, which propagate only on the 10-dimensional boundaries of the target space. The membrane solutions we construct preserve half the supersymmetries. They carry electric charge and current with respect to the gauge fields, whose generators are in the Cartan subalgebra of the two $E_{8}$ gauge groups present at the boundaries.
\end{abstract}




\section{Introduction}

M-theory, whose formulation is still unknown, is described in the low energy limit by 11-dimensional supergravity. In addition, we know that M-theory possesses membrane solutions. They appear as solutions of the equations of motion of the low energy limit of M-theory. Such solutions, in the standard case where the 11-dimensional target space has no boundaries, have been constructed in [1]. In order for these solutions to be consistent, one must couple the 11-dimensional supergravity action to a membrane action. This will guarantee that the solution exists everywhere. These solutions can describe single or multi-membrane configurations, and are constructed in much the same way as single or multi-string solutions in string theories [2]. However, although charged (with respect to gauge fields) macroscopic string solutions where constructed by Sen [3] using the techniques of [4], there are no known charged membrane solutions in 11-dimensional target spaces with boundaries.

In [5], 11-dimensional supergravity on a target space with 10-dimensional boundaries was considered. In particular, it was argued that 10-dimensional gravitational anomalies appear at the boundaries. In order to cancel these anomalies, it is necessary to introduce 248 vector multiplets in the adjoint representation of $E_{8}$ propagating on each boundary, to cancel both gravitational and gauge anomalies (the latter arising from the presence of the 496 vector multiplets). Then the low energy effective action, which is anomaly free, is 11-dimensional supergravity coupled to 10-dimensional super YangMills.

In [6], it was suggested that open membrane solutions should exists in 11dimensional supergravity on such a target space with 10-dimensional boundaries. The important requirement that open membranes must end on boundaries [7], something that is not required of open strings, is readily satisfied by demanding that the open membrane solutions stretch between the two 10-dimensional boundaries. The source of the open membrane solution is described by a 3-dimensional super-membrane action. At the boundaries, this action becomes 2-dimensional and therefore allows for 2-dimensional gravitational anomalies. In order to cancel such anomalies, it is necessary to introduce current algebras with central charge $c=8$ at each boundary. Thus the anomaly free action for the super-membrane has two terms; the first term describes the propagation of the membrane on the target space, while the 
second term couples the boundaries of the membrane to $c=8$ current algebras which are believed to generate, in the low energy limit, the 496 vector multiplets.

The action for the current algebra can always be written, after making use of Wilson lines, in terms of the Cartan subalgebra of each $E_{8}$ gauge group. It will be this form of the current algebra that will be most important to us. In finding open membrane solutions, we must consider the 11-dimensional supergravity action with a target space having two 10-dimensional boundaries. This action must then couple to the open membrane action described in the previous paragraph. This will guarantee that the membrane solution exists everywhere. Our purpose in this paper is to present explicit open membrane solutions which are charged with respect to the gauge fields, whose generators are in the Cartan subalgebra of the two $E_{8}$ gauge groups. The paper is divided as follows. In Section 2 we present the action, which will support charged open membrane solutions, and we give our conventions. In Section 3 we present our anzats and verify that half the supersymmetries are preserved. We show that the equations of motion obtained from the action are satisfied for our charged open membrane solution. We also construct a charged multi-open-membrane solution. In Section 4 we give our concluding remarks. We discuss the type IA macroscopic string obtained from dimensional reduction of the open membrane solution, and we comment on the relation of our membrane solution to the uncharged membrane of Duff and Stelle [1] and to the charged macroscopic string solution found by Sen [3].

\section{Action and Conventions}

The bosonic sector of 11-dimensional supergravity on $R^{10} \times S^{1} / Z_{2}$ is $[5$

$$
\begin{aligned}
S_{11}= & -\frac{1}{\kappa^{2}} \int_{M^{11}} d^{11} x \sqrt{-g}\left(-\frac{1}{2} R+\frac{1}{48} G_{M N O P} G^{M N O P}\right) \\
& -\frac{\sqrt{2}}{3456 \kappa^{2}} \int_{M^{11}} G \wedge G \wedge C-\frac{1}{4 \lambda^{2}} \int_{\partial M^{11}} d^{10} x \sqrt{-h} \operatorname{tr}\left(F_{A B}^{I} F^{I A B}\right),
\end{aligned}
$$

where $\partial M^{11}$ is a copy of $M^{10}$ for each boundary. In the first term of $(1), g$ is the 11-dimensional metric and $C$ is the 3 -form potential whose 4 -form field strength is $G$. In the last term, $A^{I}(I=1, \ldots, 248)$ are the gauge potentials

filling the adjoint of $E_{8}$, whose 2-form field strengths are denoted by $F^{I}$. The 
metric $h$ in the last term, which couples to the gauge fields, is the restriction of the 11-dimensional metric to each boundary, defined by each of the fixed points of the interval $I \simeq S^{1} / Z_{2}$. We thus use indices $(M, N, O, P, .$.$) for$ the 11-dimensional metric but indices $(A, B, C, D, .$.$) for the 10-dimensional$ metric, and our notations and conventions generally follow [5]. In particular, the 4 -form field strength is given by

$$
\begin{aligned}
G_{z A B C} & =\left(\partial_{z} C_{A B C} \pm 23 \text { terms }\right)+\frac{\kappa^{2}}{\sqrt{2} \lambda^{2}} \delta(z) \omega_{A B C} \\
G_{A B C D} & =\partial_{A} C_{B C D} \pm 23 \text { terms }
\end{aligned}
$$

where $z=x^{11}$ and $\omega_{A B C}$ is the Chern-Simons term

$$
\omega_{A B C}=\operatorname{tr}\left(A_{A} F_{B C}+\frac{2}{3} A_{A}\left[A_{B}, A_{C}\right]+\text { cyclic permutations }\right) .
$$

The second term in $G_{z A B C}$ is included to solve the modified Bianchi identity, as explained in [5].

Concerning the gauge potentials, we introduce Wilson lines and break each $E_{8}$ down to $U(1)^{8}$, and we will only consider this gauge group for the remainder of the article. The open membrane action which couples to (1) is then

$$
S_{o m}=S_{m}+\mu \int_{\partial M^{3}} d^{2} \zeta \epsilon^{i j} \partial_{i} Y^{I} A_{A}^{I} \partial_{j} X^{A}
$$

where

$$
S_{m}=-T \int_{M^{3}} d^{3} \zeta\left(\sqrt{-\gamma}-\sqrt{2} \epsilon^{i j k} \partial_{i} X^{M} \partial_{j} X^{N} \partial_{k} X^{P} C_{M N P}\right)
$$

In (4), $\partial M^{3}$ is a copy of $S^{1} \times R$ for each boundary. Also, there are 8 internal coordinates $Y^{I}$ at each boundary, obtained by bosonization of the left-handed Majorana fermions generating the $E_{8}$ current algebra, fulfilling

$$
\left(\sqrt{-\eta} \eta^{i j}-\epsilon^{i j}\right) \partial_{i} Y^{I}=0
$$

where the metric $\eta_{i j}$ is the restriction of the $3-\mathrm{D}$ induced metric $\gamma_{i j}$ on the world-volume of the membrane, to the 2-D boundary of the membrane.

The action we shall use to construct the open membrane solutions is

$$
S=S_{11}+S_{o m}
$$




\section{The Charged Open Membrane Solution}

We introduce the following ansatz for the metric,

$$
\begin{aligned}
d s^{2} & =-\left(e^{2 A(r)}-2 B(z, r) e^{A(r)}\right) d t^{2}+\left(e^{2 A(r)}+2 B(z, r) e^{A(r)}\right) d x^{2} \\
& +4 B(z, r) e^{A(r)} d x d t+e^{2 A(r)} d z^{2}+e^{2 Q(r)} \delta_{n m} d x^{n} d x^{m}
\end{aligned}
$$

and three-form potential

$$
C_{t x z}=e^{C(r)} .
$$

For the gauge potentials we use the ansatz

$$
A_{t}^{I}=A_{x}^{I}=\alpha^{I} e^{C(r)}
$$

where the $\alpha^{I}$ 's are constants. Here the index $t$ labels the 11-dimensional timelike coordinate, $x$ is a spacelike coordinate along the membrane and parallel to the boundary, $z$ is the spacelike coordinate also along the membrane, but transverse to the boundary (already introduced in (2)). The remaining eight coordinates are labeled by indices $(m, n, p, .$.$) and are all transverse to the$ membrane but parallel to the boundary, and as usual, the radial coordinate $r$ is defined by $r^{2}=\delta_{n m} x^{n} x^{m}$.

Supersymmetry for a bosonic membrane requires that away from the boundaries the variation of the gravitino vanishes, while at the boundary, the variation of both the gravitino and the gaugino vanish. These conditions can be summarized as follows

$$
\begin{aligned}
\delta \lambda^{I} & =F_{A B}^{I} \Gamma^{A B} \epsilon=0, \\
\delta \psi_{M} & =\left(\partial_{M}+\frac{1}{4} \omega_{M}{ }^{\bar{M} \bar{N}} \Gamma_{\bar{M} \bar{N}}+\frac{\sqrt{2}}{288}\left(\Gamma_{M}^{P Q R S}{ }_{M}+8 \Gamma^{P Q R} \delta_{M}^{S}\right) G_{P Q R S}\right) \epsilon=0
\end{aligned}
$$

where $(\bar{M}, \bar{N})$ are 11-D tangent space indices (flat indices).

The first condition in (11), which requires the variation of the gaugino to vanish, can be satisfied for our ansatz provided the spinor $\epsilon$ satisfies

$$
\left(\Gamma^{t}+\Gamma^{x}\right) \epsilon=0
$$

at the boundary. The non vanishing elfbeins corresponding to the metric (8) are

$$
\begin{aligned}
& E_{t}^{\bar{t}}=-e^{A}+B, \quad E_{x}^{\bar{x}}=e^{A}+B, \quad E_{x}^{\bar{t}}=E_{t}^{\bar{x}}=B, \\
& E_{m}^{\bar{n}}=\delta_{m}^{\bar{n}} e^{Q}, \quad E_{z}^{\bar{z}}=e^{A} .
\end{aligned}
$$


Then, from (12), it follows that

$$
\left(\Gamma_{\bar{t}}+\Gamma_{\bar{x}}\right) \epsilon=0 .
$$

This condition is equivalent to requiring that the spinor be chiral in the 10-D sense, and we have the same situation as encountered by Sen [3].

Next we consider the gravitino variations. Following Duff and Stelle [唒, we decompose the gamma-matrices in the following way

$$
\Gamma_{\bar{M}}=\left(\gamma_{\bar{\alpha}} \otimes \Gamma_{9}, 1 \otimes \Sigma_{\bar{a}}\right),
$$

where $\gamma_{\bar{\alpha}}$ and $\Sigma_{\bar{a}}$ are the $\mathrm{D}=3$ and $\mathrm{D}=8$ (flat) Dirac matrices, respectively, while $\Gamma_{9} \equiv \Sigma_{\overline{4}} \Sigma_{\overline{5}} \ldots \Sigma_{\overline{1}}$, where the bar denotes tangent space indices (flat indices).

We shall also use extensively the picture that spacetime $R^{10} \times S^{1} / Z_{2}$ can be considered a manifold with boundaries. In the following we consider for simplicity only one of the boundaries. Let it be defined by $z=0$, and let the bulk-part of spacetime correspond to $z<0$. The function $B(z, r)$, as introduced in the metric (8), is now decomposed as follows

$$
B(z, r)=f(r)(z+|z|),
$$

so that $B(z, r)$ vanishes everywhere in bulk and at the boundary, but its $z$-derivatives survive at the boundary. Now using (16), as well as (12) at the boundary, the gravitino variations reduce for $z \leq 0$ to

$$
\begin{aligned}
\delta \psi_{t} & =\left(\partial_{t}+\frac{1}{2} \gamma_{t} \Sigma^{m} A_{, m} \Gamma_{9}+\sqrt{2} \gamma_{t} e^{-3 A+C} \Sigma^{m} C_{, m}\right) \epsilon \\
\delta \psi_{x} & =\left(\partial_{x}+\frac{1}{2} \gamma_{x} \Sigma^{m} A_{, m} \Gamma_{9}+\sqrt{2} \gamma_{x} e^{-3 A+C} \Sigma^{m} C_{, m}\right) \epsilon \\
\delta \psi_{z} & =\left(\partial_{z}+\frac{1}{2} \gamma_{z} \Sigma^{m} A_{, m} \Gamma_{9}+\sqrt{2} \gamma_{z} e^{-3 A+C} \Sigma^{m} C_{, m}\right) \epsilon \\
\delta \psi_{m} & =\left(\partial_{m}-\frac{1}{4}\left(\Sigma_{m} \Sigma^{n}-\Sigma^{n} \Sigma_{m}\right) Q_{, n}-\sqrt{2} e^{-3 A+C} C_{, m} \Gamma_{9}\right. \\
& \left.+\frac{\sqrt{2}}{4} e^{-3 A+C}\left(\Sigma_{m} \Sigma^{n}-\Sigma^{n} \Sigma_{m}\right) C_{, n} \Gamma_{9}\right) \epsilon .
\end{aligned}
$$

Notice that we have skipped terms automatically vanishing for $z \leq 0$ (but generally not vanishing outside spacetime, corresponding to $z>0$ ) due to 
(16), as well as terms multiplying $\left(\Gamma^{t}+\Gamma^{x}\right)$, c.f. (12). Then if

$$
\begin{aligned}
& C=3 A-\log (6 \sqrt{2}), \\
& Q=-A / 2,
\end{aligned}
$$

and provided that

$$
\epsilon=e^{-A / 2} \epsilon_{o},
$$

where the constant spinor $\epsilon_{o}$ satisfies

$$
\left(1+\Gamma_{9}\right) \epsilon_{o}=0
$$

we find that all supersymmetry variations of the gravitino and gaugino vanish in bulk $(z<0)$ and at the boundary $(z=0)$. Notice that the two conditions $\left(1+\Gamma_{9}\right) \epsilon=0$ and $\left(\Gamma^{t}+\Gamma^{x}\right) \epsilon=0$ are equivalent since巴

$$
\Gamma_{\bar{z}} \epsilon=\epsilon \quad \text { and } \quad \Gamma_{9} \Gamma_{\bar{t}} \Gamma_{\bar{x}} \Gamma_{\bar{z}}=-1
$$

on $R^{10} \times S^{1} / Z_{2}$, [6]. The conditions obtained for the spinor thus correspond to breaking half the supersymmetries in bulk and at the boundary.

Using the following embedding for the membrane

$$
X^{\mu}(\zeta)=\zeta^{\mu}, \mu=t, x, z \text { and } X^{m}(\zeta)=\text { const. }
$$

the equation of motion for the G-field, for our ansatz, is

$$
\partial_{m}\left(\sqrt{-g} G^{m t x z}\right)=-\sqrt{2} T \kappa^{2} \delta^{8}(r),
$$

taking the location of the core of the membrane to be at $r=0$. This equation is solved by

$$
A(r)=-\frac{1}{3} \log \left(1+\frac{K}{r^{6}}\right)
$$

provided the constant $K$ is related to the membrane tension by

$$
K=\frac{T \kappa^{2}}{3 \Omega_{8}}
$$

where $\Omega_{8}$ is the area of the unit sphere in $R^{8}$.

\footnotetext{
${ }^{1}$ We thank P. Horava for a clarifying comment to us about this point.
} 
In addition we find, for our ansatz and the embedding (22), that the equations of motion for the gauge fields are

$$
\partial_{n}\left(\sqrt{-h} F^{I n \mu}\right)+\sqrt{2} \sqrt{-g} G^{n \nu \mu z} F_{n \nu}^{I}=\mu \lambda^{2} \epsilon^{\rho \nu} \partial_{\rho} Y^{I} \partial_{\nu} X^{\mu} \delta^{8}(r),
$$

where $\mu, \nu, \rho, .$. are either $t$ or $x$. Notice that the gauge-dependent term, which arises when varying the Chern-Simons term, has been omitted, c.f. [3]. This equation is solved by (24), (10) and (9) provided

$$
Y^{I}=\frac{K \alpha^{I} \Omega_{8}}{\sqrt{2} \mu \lambda^{2}} e^{3 A}(t+x),
$$

where the function $A$ is evaluated at $X^{m}=$ const., c.f. (22). Notice also that (27) is consistent with (6), as it should be.

Next we consider the Einstein equations

$$
R_{M N}-\frac{1}{2} g_{M N} R=T_{M N}
$$

For completeness we list below the non-vanishing components of the Riccitensor and the energy-momentum tensor

$$
\begin{aligned}
R_{z z} & =-e^{3 A} \delta^{n m} A_{, n m} \\
R_{n m} & =\frac{1}{2} \delta_{n m} \delta^{p q} A_{, p q}-\frac{9}{2} A_{, n} A_{, m} \\
\left(\begin{array}{ll}
R_{t t} & R_{t x} \\
R_{x t} & R_{x x}
\end{array}\right) & =-e^{3 A} \delta^{n m} A_{, n m}\left(\begin{array}{cc}
-1 & 0 \\
0 & 1
\end{array}\right)-e^{-A} B^{\prime \prime}\left(\begin{array}{ll}
1 & 1 \\
1 & 1
\end{array}\right),
\end{aligned}
$$

so that

$$
R=e^{A}\left(\delta^{n m} A_{, n m}-\frac{9}{2} \delta^{n m} A_{, n} A_{, m}\right) .
$$

The energy-momentum tensor takes the form

$$
\begin{aligned}
T_{z z} & =-\frac{9}{4} e^{3 A} \delta^{n m} A_{, n} A_{, m}-\kappa^{2} T e^{6 A} \delta^{8}(r) \\
T_{n m} & =-\frac{9}{2} A_{, n} A_{, m}+\frac{9}{4} \delta_{n m} \delta^{p q} A_{, p} A_{, q} \\
\left(\begin{array}{cc}
T_{t t} & T_{t x} \\
T_{x t} & T_{x x}
\end{array}\right) & =-\left(\frac{9}{4} e^{3 A} \delta^{n m} A_{, n} A_{, m}+\kappa^{2} T e^{6 A} \delta^{8}(r)\right)\left(\begin{array}{cc}
-1 & 0 \\
0 & 1
\end{array}\right) \\
& +\frac{\kappa^{2}}{\lambda^{2}} \delta(z) \delta^{n m} A_{t, n}^{I} A_{t, m}^{I}\left(\begin{array}{cc}
1 & 1 \\
1 & 1
\end{array}\right),
\end{aligned}
$$


where we used that $A_{t}^{I}=A_{x}^{I}$. Again it should be stressed that we have skipped terms automatically vanishing for $z \leq 0$ (but generally not vanishing for $z>0$ ). Notice also that the $z$-dependence in the energy-momentum tensor appears only via the delta-function multiplying the Yang-Mills term. This delta-function arises because the gauge fields live only at the boundaries. Similarly, the $z$-dependence in the Einstein tensor appears only in the $t-x$ sector due to the $z$-dependence of the metric (8) in the $t-x$ sector.

It is now straightforward to check that all the Einstein equations are consistently fulfilled by our ansatz, provided the function $f(r)$, as introduced in (16), is given by

$$
f(r)=\frac{-\kappa^{2}}{2 \lambda^{2}} \delta^{n m} A_{t, n}^{I} A_{t, m}^{I} e^{A},
$$

where $A_{t}^{I}$ is given by (10), (18) and (24).

Finally we consider the equations of motion for the membrane source, as obtained from (4), (5). For our ansatz and embedding, the boundary term in (4) does not give any contribution so we are left with

$$
\begin{aligned}
\frac{1}{2} \sqrt{-\gamma} \gamma^{i j} X_{, i}^{M} X_{, j}^{N} g_{M N, P} & -\partial_{j}\left(\sqrt{-\gamma} \gamma^{i j} g_{M P} X_{, i}^{M}\right) \\
& =-\frac{\sqrt{2}}{6} \epsilon^{i j k} \partial_{i} X^{M} \partial_{j} X^{N} \partial_{k} X^{O} G_{P M N O} .
\end{aligned}
$$

Again we find that our ansats and embedding exactly fulfills the equations for $z \leq 0$. This concludes the proof that our open membrane with boundary solves all the equations for $g_{M N}, C_{M N P}, A_{A}$ as well as the equations for the membrane source itself.

Then, the open membrane solution is

$$
\begin{aligned}
d s^{2}= & -\left[\left(1+\frac{K}{r^{6}}\right)^{-\frac{2}{3}}+\frac{\kappa^{2}\left(\alpha^{I}\right)^{2} K^{2}}{2 \lambda^{2} r^{14}}(z+|z|)\left(1+\frac{K}{r^{6}}\right)^{-\frac{14}{3}}\right] d t^{2} \\
& +\left[\left(1+\frac{K}{r^{6}}\right)^{-\frac{2}{3}}-\frac{\kappa^{2}\left(\alpha^{I}\right)^{2} K^{2}}{2 \lambda^{2} r^{14}}(z+|z|)\left(1+\frac{K}{r^{6}}\right)^{-\frac{14}{3}}\right] d x^{2} \\
& -\left[\frac{\kappa^{2}\left(\alpha^{I}\right)^{2} K^{2}}{\lambda^{2} r^{14}}(z+|z|)\left(1+\frac{K}{r^{6}}\right)^{-\frac{14}{3}}\right] d x d t \\
& +\left(1+\frac{K}{r^{6}}\right)^{-\frac{2}{3}} d z^{2}+\left(1+\frac{K}{r^{6}}\right)^{\frac{1}{3}} \delta_{n m} d x^{n} d x^{m},
\end{aligned}
$$




$$
\begin{aligned}
A_{t}^{I} & =A_{x}^{I}=\frac{\alpha^{I}}{6 \sqrt{2}}\left(1+\frac{K}{r^{6}}\right)^{-1} \\
C_{t x z} & =\frac{1}{6 \sqrt{2}}\left(1+\frac{K}{r^{6}}\right)^{-1} .
\end{aligned}
$$

Before carrying on, we should pause to make a statement about the parity of the membrane solution (34). It is sometimes convenient to consider the target space to be $S^{1} \times R^{10}$ instead of $S^{1} / Z_{2} \times R^{10}$. We should then impose parity-invariance around some point $z \in S^{1}$, (say) $z=0$. Under a parity transformation

$$
z \rightarrow-z
$$

we must demand that

$$
\begin{aligned}
C_{A B C}(z) & =-C_{A B C}(-z), \\
C_{A B z}(z) & =C_{A B z}(-z), \\
g_{A B}(z) & =g_{A B}(-z), \\
g_{A z}(z) & =-g_{A z}(-z), \\
g_{z z}(z) & =g_{z z}(-z),
\end{aligned}
$$

where the indices $(A, B, C)$ are 10-dimensional. The requirements (36), are equivalent to demanding that the solutions on the target space $S^{1} \times R^{10}$ also be solutions on the target space $S^{1} / Z_{2} \times R^{10}$. It is clear from our anzats, that the metric does not satisfy all the conditions (36). However, in order to avoid this inconsistency, we should use the ansatz (16) only when the spacetime is identified with $z \leq 0$. If spacetime is instead identified with $z \geq 0$, we should use instead of (16) the ansatz $B(z, r)=f(r)(-z+|z|)$. In this way, the metric $g_{A B}$ actually is, by definition, even under $z \rightarrow-z$, and the only effect of the " $|z| "$ is to produce a non-vanishing $z$-derivative at $z=0$, which is necessary in the Einstein tensor, as explained after equation (31). However, for our purposes here it is generally most convenient to actually think of an 11-dimensional spacetime with boundaries.

We must now show that the membrane source (22) also preserves half the supersymmetries. Following [8], we must require for a bosonic membrane source that the Killing spinor also satisfies

$$
(1+\Gamma) \epsilon=0
$$


where

$$
\Gamma \equiv \frac{1}{3 ! \sqrt{-\gamma}} \epsilon^{i j k} \partial_{i} X^{M} \partial_{j} X^{N} \partial_{k} X^{P} \Gamma_{M N P} .
$$

Using (15), we find that $\Gamma=-\Gamma_{\bar{t}} \Gamma_{\bar{x}} \Gamma_{\bar{z}}$ and then using (21), the condition (37) reduces to

$$
\left(1+\Gamma_{9}\right) \epsilon=0,
$$

that is, equation (20). Hence we are able to satisfy (37) in the same manner as done in [1]. At the boundaries we should strictly speaking use instead of (38) the expression

$$
\Gamma \equiv \frac{1}{2 ! \sqrt{-\eta}} \epsilon^{i j} \partial_{i} X^{M} \partial_{j} X^{N} \Gamma_{M N} .
$$

Then we get $\Gamma=-\Gamma_{\bar{t}} \Gamma_{\bar{x}}$, which from (21) again reduces to (39) when acting on the spinor.

The charges and currents of the membrane solution can be read off from the 2-form field strength and the 4 -form field strength in the limit that $r \rightarrow \infty$

$$
\begin{aligned}
F_{x r}^{I} & =F_{t r}^{I}=\frac{K \alpha^{I}}{\sqrt{2} r^{7}}\left(1+\frac{K}{r^{6}}\right)^{-2} \sim \frac{K \alpha^{I}}{\sqrt{2} r^{7}}, \\
G_{t x z r} & =\frac{6 K}{\sqrt{2} r^{7}}\left(1+\frac{K}{r^{6}}\right)^{-2} \sim \frac{6 K}{\sqrt{2} r^{7}} .
\end{aligned}
$$

Using the standard formula for a 1-brane in 10 dimensions, we conclude that the $U(1)$-charges and currents at the boundary are (up to a numerical factor)

$$
Q^{I}=J^{I}=K \alpha^{I} .
$$

Similarly, using the standard formula for a 2-brane in 11 dimensions, we conclude that the "axion-charge" is (up to a numerical factor)

$$
Z=K \text {. }
$$

Although we have only constructed a single open membrane solution, it is straightforward to show that a stable multi-membrane solution is obtained by a linear superposition of solutions

$$
A(\vec{r})=-\frac{1}{3} \log \left(1+\sum_{i} \frac{K}{\left|\vec{r}-\vec{r}_{i}\right|^{6}}\right),
$$


where $\vec{r}_{i}$ denotes the location of the core of the $i^{\prime} t h$ membrane, together with the corresponding generalization of the embedding (22). This is the typical situation in soliton theory, so we shall not go into more details here. The multi-membrane solution takes the form (34) after the substitution

$$
\left(1+\frac{K}{r^{6}}\right) \longrightarrow\left(1+\sum_{i} \frac{K}{\left|\vec{r}-\vec{r}_{i}\right|^{6}}\right)
$$

that is

$$
\begin{aligned}
d s^{2}= & -\left[\left(1+\sum_{i} \frac{K}{\left|\vec{r}-\overrightarrow{r_{i}}\right|^{6}}\right)^{-\frac{2}{3}}+\frac{\kappa^{2}\left(\alpha^{I}\right)^{2} K^{2}}{2 \lambda^{2} r^{14}}(z+|z|)\left(1+\sum_{i} \frac{K}{\left.\left.\mid \vec{r}-\overrightarrow{\left.r_{i}\right|^{6}}\right)^{-\frac{14}{3}}\right] d t^{2}}\right.\right. \\
& +\left[\left(1+\sum_{i} \frac{K}{\left|\vec{r}-\overrightarrow{r_{i}}\right|^{6}}\right)^{-\frac{2}{3}}-\frac{\kappa^{2}\left(\alpha^{I}\right)^{2} K^{2}}{2 \lambda^{2} r^{14}}(z+|z|)\left(1+\sum_{i} \frac{K}{\mid \vec{r}-\overrightarrow{\left.r_{i}\right|^{6}}}\right)^{-\frac{14}{3}}\right] d x^{2} \\
& -\left[\frac{\kappa^{2}\left(\alpha^{I}\right)^{2} K^{2}}{\lambda^{2} r^{14}}(z+|z|)\left(1+\sum_{i} \frac{K}{\left|\vec{r}-\overrightarrow{r_{i}}\right|^{6}}\right)^{-\frac{14}{3}}\right] d x d t \\
& +\left(1+\sum_{i} \frac{K}{\left|\vec{r}-\overrightarrow{r_{i}}\right|^{6}}\right)^{-\frac{2}{3}} d z^{2}+\left(1+\sum_{i} \frac{K}{\left|\vec{r}-\overrightarrow{r_{i}}\right|^{6}}\right)^{\frac{1}{3}} \delta_{n m} d x^{n} d x^{m} \\
A_{t}^{I}= & A_{x}^{I}=\frac{\alpha^{I}}{6 \sqrt{2}}\left(1+\sum_{i} \frac{K}{\left|\vec{r}-\overrightarrow{r_{i}}\right|^{6}}\right)^{-1} \\
C_{t x z}= & \frac{1}{6 \sqrt{2}}\left(1+\sum_{i} \frac{K}{\left|\vec{r}-\overrightarrow{r_{i}}\right|^{6}}\right)^{-1} .
\end{aligned}
$$

and the charges and currents of each membrane in the multi-membrane solution equal those of the single membrane solution.

\section{Concluding Remarks}

In conclusion, we have constructed open single and multi-membrane solutions of the low energy limit of M-theory on the orbifold $R^{10} \times S^{1} / Z_{2}$. These membrane solutions were shown to preserve half the supersymmetries. They carry "axion-charge" in the 11-dimensional spacetime as well as electric charge and current with respect to the gauge fields, whose generators are in the Cartan 
subalgebra of the two $E_{8}$ gauge groups present at the 10-dimensional boundaries.

There are in principle different types of dimensional reductions that can be performed on our membrane solution. Let us consider now the orbifold $R^{9} \times S^{1} \times S^{1} / Z_{2}$ and let $x$ be the coordinate on $S^{1}$. When dimensionally reducing along the $x$-direction, the resultant supergravity action is that of type IA supergravity [6]. The resultant source action is that of the type IA string which couples to $S O(16) \times S O(16)$ Chan Paton factors [9]. It turns out that the dimensional reduction of the open membrane solution is the open string solution with Chan Paton factors generated by dimensional reduction of the term which couples the gauge fields to the membrane in the source term. Thus this solution is macroscopic type IA string solution.

When performing a standard Kaluza-Klein dimensional reduction along the $z$-direction, the resultant supergravity action is the low energy limit of the heterotic string theory. However, at the present stage, it is not clear how to perform the corresponding dimensional reduction of our membrane solution. The reason is that the solution depends explicitly on the $z$-coordinate (even in a discontinuous way), so the standard ansatz for dimensional reduction does not work here. But notice that our membrane solution shows several similarities with the string solution found by Sen [3], thus we expect that our solution should actually reduce to Sens solution after a dimensional reduction along the $z$-direction. Then our solution would naturally interpolate between the membrane solution of Duff and Stelle [1] in bulk and the charged string solution of Sen [3] at the boundaries.

\section{Acknowledgements:}

We would like to thank H. Balasin, N. Kaloper and specially R. Myers for helpfull discussions. The work of F. Aldabe was supported by NSERC, Canada. A.L. Larsen was partly supported by NSERC, Canada and partly by CNRS, France. 


\section{References}

[1] M.J. Duff and K.S. Stelle, Multimembrane Solutions Of D=11 Supergravity , Phys. Lett. B253 (1991) 113.

[2] A. Dabholkar, G.W. Gibbons, J.A. Harvey and F. Ruiz-Ruiz, Superstrings And Solitons, Nucl. Phys. B340 (1990) 33.

[3] A. Sen, Macroscopic Charged Heterotic String, Nucl. Phys. B388 (1992) 457.

[4] S.F. Hassan and A. Sen, Twisting Classical Solutions In The Heterotic String Theory, Nucl. Phys. B375 (1992) 103.

[5] P. Horava and E. Witten, Eleven-Dimensional Supergravity on A Manifold with Boundary, Nucl. Phys. B475 (1996) 94.

[6] P. Horava and E. Witten, Heterotic and Type I String Dynamics ¿From Eleven Dimensions, Nucl. Phys. B460 (1996) 506.

[7] A. Strominger, Open p-Branes, Phys. Lett. B383 (1996) 44.

[8] E. Bergshoeff, E. Sezgin and P.K. Townsend, Supermembranes And Eleven-Dimensional Supergravity, Phys. Lett. B189 (1987) 75.

[9] F. Aldabe, Heterotic and Type I Strings from Twisted Supermembranes, Nucl. Phys. B473 (1996) 63. 